\title{
On the irreducibility of cones of 3-secant planes
}

\author{
Ciro Ciliberto ${ }^{1}$
}

Received: 26 May 2020 / Accepted: 4 June 2020 / Published online: 26 July 2020

(c) The Author(s) 2020

\begin{abstract}
In this paper we prove that if $X \subset \mathbb{P}^{r}$ is a 2-smooth, irreducible, projective non-degenerate variety of dimension $n$ such that $\operatorname{Sec}_{2}(X)=\mathbb{P}^{r}$, if $n>\frac{4}{7}(r-2)$ and if $X^{\prime}$ is the projection of $X$ to $\mathbb{P}^{r-1}$ from a general point, then the set of length 3 subschemes of $X^{\prime}$ which lie on a line form an irreducible variety.
\end{abstract}

\section{Introduction}

In Theorem 2 of the paper [4] Lopez and Ran prove that, given a smooth, irreducible, projective, non-degenerate variety $X \subset \mathbb{P}^{r}$ of dimension $n>\frac{2}{3}(r-1)$ whose secant variety fills up $\mathbb{P}^{r}$, the double locus (in $X$ ) of its general projection to $\mathbb{P}^{r-1}$ is irreducible. The present paper is devoted to prove an extension of this theorem. To state our result we need some preliminary.

Let $X \subset \mathbb{P}^{r}$ be as above. If $k \leq r$ is a positive integer, $X$ is said to be $k$-smooth if it is smooth and for any subscheme $Z$ of length $k+1$ of $X$, one has $h^{0}\left(\mathbb{P}^{r}, \mathcal{J}_{Z, \mathbb{P} r}(1)\right)=r-k$, or, in other words, $Z$ imposes independent conditions to the hyperplanes of $\mathbb{P}^{r}$, i.e., $Z$ spans a linear space of dimension $k$. In particular 1-smooth means smooth.

Let $X \subset \mathbb{P}^{r}$ as above be $k$-smooth. Consider the $k$-secant variety $\operatorname{Sec}_{k}(X)$ of $X$, i.e., the Zariski closure of the union all linear subspaces of dimension $k$ which are $(k+1)$-secant to $X$ at distinct, hence linearly independent, points. One sets $\operatorname{Sec}_{1}(X):=\operatorname{Sec}(X)$. Suppose $\operatorname{Sec}_{k}(X)=\mathbb{P}^{r}$. One says that $X$ has the irreducible $(k+1)$-secant cones property ((ISC) $k$ property in brief) if, given a general point $p \in \mathbb{P}^{r}$, the set of linear subspaces of dimension $k$ which are $(k+1)$-secant to $X$ passing through $p$ is irreducible. If $X$ has the (ISC) $k$ property, then for the projection $X^{\prime}$ of $X$ to $\mathbb{P}^{r-1}$ from a general point, the set of subschemes of $X^{\prime}$ of length $k+1$ which fail to impose independent conditions to the hyperplanes in $\mathbb{P}^{r-1}$ form an irreducible variety.

Our result is the following:

Ciro Ciliberto

cilibert@mat.uniroma2.it

1 Dipartimento di Matematica, Università di Roma Tor Vergata, Via della Ricerca Scientifica, 00173

Rome, Italy 
Theorem 1.1 Let $X \subset \mathbb{P}^{r}$ be a 2-smooth, irreducible, projective non-degenerate variety of dimension $n$ such that $\operatorname{Sec}_{2}(X)=\mathbb{P}^{r}$. If

$$
n>\frac{4}{7}(r-2) \text {, }
$$

then $X$ has the (ISC) 2 property.

The proof closely follows for a great part, with only very few differences, the one of Theorem 2 in [4]. One of the differences is that, in a delicate point of the argument, it is necessary to apply the Infinitesimal Terracini's Lemma from [5] (see also [2]), which plays an important role in the final part of the proof.

The hypothesis (1) is very likely not to be the sharpest one. Better bounds could be obtained if one were able to bound from below the dimension of secant varieties of varieties which, though not necessarily smooth, do not contain length 3 subschemes spanning lines (like, for example, the $n$-Veronese images of given varieties, with $n \geq 2$ ).

Of course one might conjecture a more general result. Namely:

Conjecture 1.2 Let $X \subset \mathbb{P}^{r}$ be a $k$-smooth, irreducible, projective non-degenerate variety of dimension $n$ such that $\operatorname{Sec}_{k}(X)=\mathbb{P}^{r}$. Under suitable numerical conditions involving $n$ and $r, X$ has the (ISC) $k$ property.

Unfortunately I am still unable to prove this. One of the reasons is that the infinitesimal Terracini's lemma has only been proved for $\operatorname{Sec}(X)$, whereas one needs a suitable version of it for higher secant varieties.

In this paper I will work over an algebraically closed field of characteristic zero.

\section{The proof of Theorem 1.1}

Let $(p, q, r) \in X^{3}$ be such that $p, q, r$ are distinct. By the 2-smoothness property, the span $\langle p, q, r\rangle$ is a plane. Let us consider the normalization $X^{[3]}$ of the blowing-up of $X^{3}$ along the main diagonals, with exceptional divisor $E$. A general point of an irreducible component of $E$ can be identified with a pair $(p, \zeta)$, where $p \in X$ and $\zeta$ is a length 2 subscheme of $X$ supported at a point $q \in X$ different from $p$. By the 2-smoothness hypothesis, the span $\langle p, \zeta\rangle$ is still a plane.

There is an obvious tautological $\mathbb{P}^{2}$-bundle $\pi: \mathcal{X} \rightarrow X^{[3]}$, and $\mathcal{X}$ is normal since so is $X^{[3]}$. Moreover we have a natural surjective morphism $f: \mathcal{X} \rightarrow \mathbb{P}^{r}$. The statement of Theorem 1.1 is equivalent to say that the general fibre of $f$ is irreducible.

Let us consider the Stein factorization of $f$

$$
\mathcal{X} \stackrel{h}{\longrightarrow} \mathcal{Z} \stackrel{g}{\longrightarrow} \mathbb{P}^{r}
$$

so that $\mathcal{Z}$ is normal and $g$ is generically finite and surjective. Since $\mathcal{X}$ is normal, also the generic fibre of $f$ is normal, hence the irreducible components and the connected components of that fibre coincide (see the argument at the beginning of the proof of Theorem 2 in [4]). So we need to prove that $g$ is birational. We argue by contradiction and assume this is not the case.

We may assume there is a dense open subset $U \subset \mathbb{P}^{r}$, such that $\mathbb{P}^{r}-U$ has codimension $c>1, g^{-1}(U)$ is smooth and $g^{-1}(U) \stackrel{g}{\longrightarrow} U$ is finite of degree $d>1$. Since $U$ is simply connected, as well as $\mathbb{P}^{r}$, then $g$ is ramified in codimension 1 , hence there is some irreducible 
divisor $R$ in $\mathcal{Z}$ (with non-empty intersection with $g^{-1}(U)$ ), such that $g$ is ramified along $R$. Set $F:=h^{-1}(R)$. This is an irreducible divisor of $\mathcal{X}$, such that $f$ is ramified there, i.e., the differential of $f$ drops rank, along $F$.

Assume first that $F$ is not contained in any component of $E$. Then a general point of $F$ can be identified with a quadruple $(p, q, r, t)$, with $p, q, r \in X$ distinct (hence independent) points and $t \in\langle p, q, r\rangle$ general. By Terracini's Lemma one has

$$
\operatorname{Im}_{\mathbb{P}}\left(d f_{(p, q, r, t)}\right)=\left\langle T_{X, p}, T_{X, q}, T_{X, r}\right\rangle,
$$

which is independent of $t \in\langle p, q, r\rangle$ (here $T_{X, x}$ denotes the projective tangent space to $X$ at $x$ and by $\operatorname{Im}_{\mathbb{P}}\left(d f_{(p, q, r, t)}\right)$ we mean the projective closure of $\left.\operatorname{Im}\left(d f_{(p, q, r, t)}\right)\right)$. Hence $F$ is the pull back of an irreducible divisor $D$ of $X^{[3]}$. Moreover one has

$$
\rho:=\operatorname{dim}\left(\operatorname{Im}_{\mathbb{P}}\left(d f_{(p, q, r, t)}\right)\right)=\operatorname{dim}\left(\left\langle T_{X, p}, T_{X, q}, T_{X, r}\right\rangle\right)<r .
$$

Note that we have the three projection maps $p_{i}: X^{[3]} \rightarrow X$, with $i=1,2,3$, to the three factors of $X^{3}$. Assume first that the restriction of each one of the maps $p_{i}$ to $D$ is surjective onto $X$, for $i=1,2,3$. Fix $x, y \in X$ general points and consider

$$
D_{x, y}:=p_{1}\left(p_{2}^{-1}(x) \cap p_{3}^{-1}(y) \cap D\right)
$$

which is a divisor on $X$. Take a general point $z \in D_{x, y}$ which is a general point of $X$. Set

$$
W:=\left\langle T_{X, x}, T_{X, y}\right\rangle
$$

and note that, by Terracini's Lemma, one has

$$
s:=\operatorname{dim}(W)=\operatorname{dim}(\operatorname{Sec}(X)) .
$$

One has

$$
\rho-1 \leq \operatorname{dim}\left(\left\langle T_{D_{x, y}, z}, W\right\rangle\right) \leq \rho .
$$

Now consider the following diagram

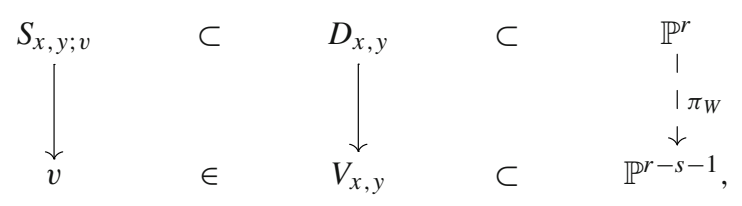

where $\pi_{W}$ is the projection from the subspace $W, V_{x, y}$ is the image of $D_{x . y}, v \in V_{x, y}$ is a general point (in a component of $V_{x, y}$ ) and $S_{x, y ; v}$ is the fibre of $D_{x . y} \stackrel{\pi_{W}}{\longrightarrow} V_{x, y}$ over $v$.

One has

$$
\rho-s-2 \leq \operatorname{dim}\left(V_{x, y}\right) \leq \rho-s-1
$$

and accordingly

$$
n+s-\rho+1 \geq \operatorname{dim}\left(S_{x, y ; v}\right) \geq n+s-\rho .
$$

Suppose the right hand side values hold in (2) and (3). This implies that, for $z \in D_{x, y}$ general (in a component), one has

$$
\left\langle T_{D_{x, y}, z}, W\right\rangle=\left\langle T_{X, z}, W\right\rangle
$$


and this space projects down from $W$ to $T_{V_{x, y}, v}$, with $v=\pi_{W}(z)$. Hence

$$
T_{X, z} \subseteq T:=\left\langle T_{V_{x, y}, v}, W\right\rangle .
$$

Note that $\operatorname{dim}(T)=\operatorname{dim}\left(T_{V_{x, y}, v}\right)+\operatorname{dim}(W)+1=\rho$ and $T$ is tangent to $X$ along $S_{x, y ; v}$. Then we can apply the extension of Zak's Theorem on Tangencies contained in [1, Theorem 6.1], obtaining

$$
\rho \geq \operatorname{dim}(X)+\operatorname{dim}\left(\operatorname{Sec}\left(S_{x, y ; v}\right)\right)=n+\operatorname{dim}\left(\operatorname{Sec}\left(S_{x, y ; v}\right)\right) .
$$

One has $\operatorname{dim}\left(S_{x, y ; v}\right)=n+s-\rho>0$. In fact, otherwise we would have $n+s=\rho$, which implies that $T_{X, z}$ intersects $W$ in one point. Therefore $T_{X, z} \cap T_{X, x}$ and $T_{X, z} \cap T_{X, y}$ can consist of at most one point, and by generality also $T_{X, x} \cap T_{X, y}$ can be at most one point. This implies that $s \geq 2 n$ and therefore $r>\rho=n+s \geq 3 n$, which contradicts (1). Since $S_{x, y ; v}$ is not a projective space because $X$ is 2 -smooth, we have

$$
\operatorname{dim}\left(\operatorname{Sec}\left(S_{x, y ; v}\right)\right) \geq \operatorname{dim}\left(S_{x, y ; v}\right)+1 \geq n+s-\rho+1 .
$$

Plugging in (4), taking into account that $\rho \leq r-1$ and that, by Zak's Theorem on linear normality one has

$$
s=\operatorname{dim}(\operatorname{Sec}(X)) \geq \frac{3}{2} n+1,
$$

we get

$$
r \geq \frac{7}{4} n+2
$$

against (1).

Suppose next that the left hand side values are attained in (2) and (3). Then for $z \in S_{x, y ; v}$ general, the projection of $T_{X, z}$ from $W$ is a linear space of dimension $\rho-s-1$ containing $T_{V_{x, y}, v}$, which has dimension $\rho-s-2$. The set of linear subspaces of dimension $\rho-s-1$ containing $T_{V_{x, y}, v}$ is a linear space of dimension $r-\rho$. This implies that we find a linear space of dimension $\rho$, i.e., the span of $W$ with $T_{X, z}$, which is tangent to $X$ along a subvariety of $S_{x, y ; v}$ of codimension at most $r-\rho$. Applying Zak's Theorem on Tangencies one has

$$
\rho \geq \operatorname{dim}(X)+\operatorname{dim}\left(S_{x, y ; v}\right)-(r-\rho),
$$

which, taking into account $\rho \leq r-1$ and (5) gives again (6), a contradiction.

Next suppose that one of the restrictions of the projections $p_{i}$ to $D$ is not surjective onto $X$, e.g., assume that $p_{1}: D \rightarrow X$ is not surjective. Let $D^{\prime}$ be its image. Then $D^{\prime}$ is a divisor of $X$ and $D$ is the proper transform on $X^{[3]}$ of $D^{\prime} \times X^{2}$, so that $p_{2}$ and $p_{3}$ restricted to $D$ are surjective onto $X$. Then $D_{x, y}=p_{1}\left(p_{2}^{-1}(x) \cap p_{3}^{-1}(y) \cap D\right)$ coincides with $D^{\prime}$, i.e., does not depend on $x$ and $y$ general on $X$. We can repeat the same argument as above. The only difference is that $z \in D^{\prime}$ is no longer general in $X$. However, this is no problem. The whole argument works in the same way, including the proof that $\operatorname{dim}\left(S_{x, y ; v}\right)=n+s-\rho>0$. In fact, if $n+s=\rho$, then again $T_{X, z}$ intersects $W$ in one point. Therefore $T_{X, z} \cap T_{X, x}$ and $T_{X, z} \cap T_{X, y}$ can consist of at most one point, and by generality of $x$ and $y$ on $X$ also $T_{X, x} \cap T_{X, y}$ can be at most one point. This implies that $s \geq 2 n$ and again $r>\rho=n+s \geq 3 n$, which contradicts (1).

Finally we have to discuss the case in which $F$ is a component of $E$. This is the part of the proof in which we need some argument different from the ones in [4]. In this case the general element of $F$ can be assumed to be of the type $(p, \zeta, z)$, where $p \in X$ is general, $\zeta$ 
is a general length 2 subscheme of $X$ supported at a general point $q \in X$ (hence different from $p$ ) and $z \in\langle p, \zeta\rangle$.

Define the linear subspace $T_{X, q, \zeta}$ in the following way: $T_{X, q, \zeta}^{\perp}$ is the set of all hyperplanes in $\mathbb{P}^{r}$ which intersect $X$ in a divisor which has at least a double point at $q$ and at least another double point at the infinitely near point to $q$ corresponding to the scheme $\zeta$. It is easy to see that

$$
\operatorname{dim}\left(T_{X, q, \zeta}\right) \leq 2 n+1
$$

Let us suppose first that

$$
\operatorname{dim}\left(T_{X, q, \zeta}\right)<2 n+1 .
$$

If $\operatorname{dim}(\operatorname{Tan}(X))<2 n$, then $\operatorname{Tan}(X)=\operatorname{Sec}(X)$ (see [3, Cor. 5.5]) and so

$$
\mathbb{P}^{r}=\operatorname{Sec}_{2}(X)=\operatorname{Join}(\operatorname{Sec}(X), X)=\operatorname{Join}(\operatorname{Tan}(X), X),
$$

which implies that $f(E)=\mathbb{P}^{r}$, so that also $f(F)=\mathbb{P}^{r}$, a contradiction. Hence one has $\operatorname{dim}(\operatorname{Tan}(X))=2 n$. Then by a result of Terracini (see [5]) which can be seen as an Infinitesimal Terracini's Lemma (see [2]), (8) implies that $s:=\operatorname{dim}(\operatorname{Sec}(X))<2 n+1$, and again $\operatorname{Tan}(X)=\operatorname{Sec}(X)$ and one gets a contradiction as above. Thus we may assume the equality holds in (7), so that, by Terracini's result in [5], one has $s=2 n+1$.

We set $W=T_{X, q, \zeta}$ and we proceed as before, projecting from $W$. The argument is similar to the one we run above.

By a suitable version of Terracini's Lemma one has

$$
\operatorname{Im}_{\mathbb{P}}\left(d f_{(p, \zeta, z)}\right)=\left\langle T_{X, p}, T_{X, q, \zeta}\right\rangle .
$$

Since this is independent of $z$, then $F$ is the pull back of a divisor $D$ of $X^{[3]}$. Moreover one has

$$
\rho:=\operatorname{dim}\left(\operatorname{Im}_{\mathbb{P}}\left(d f_{(p, \zeta, z)}\right)\right)=\operatorname{dim}\left(\left\langle T_{X, p}, T_{X, q, \zeta}\right\rangle\right)<r .
$$

The general point of $D$ can be now identified with a pair $(p, \zeta)$ as above, with $p \in X$ general and $\zeta$ a general length 2 scheme of $X$. Then one has two maps $\sigma_{1}:(p, \zeta) \in D \mapsto$ $p \in X$ and $\sigma_{2}:(p, \zeta) \in D \mapsto \zeta \in X^{(2)}$, where $X^{(2)}$ is the variety of all non-reduced schemes of length 2 on $X$. By the above considerations, both maps $\sigma_{1}$ and $\sigma_{2}$ are surjective onto their targets. If we fix a general point $\zeta \in X^{(2)}$

$$
D_{\zeta}:=\sigma_{1}\left(\sigma_{2}^{-1}(\zeta) \cap D\right)
$$

is a divisor on $X$.

Take a general point $z \in D_{\zeta}$ which is a general point of $X$. Then one has

$$
\rho-1 \leq \operatorname{dim}\left(\left\langle T_{D_{\zeta}, z}, W\right\rangle\right) \leq \rho .
$$

One has the diagram

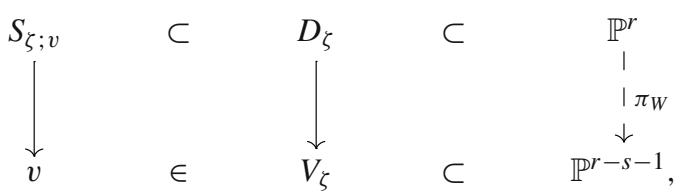


where $\pi_{W}$ is the projection from $W, V_{\zeta}$ is the image of $D_{\zeta}, v \in V_{\zeta}$ is a general point and $S_{\zeta ; v}$ is the fibre of $D_{\zeta} \stackrel{\pi_{W}}{\longrightarrow} V_{\zeta}$ over $v$.

One has

$$
\begin{aligned}
& \rho-s-2 \leq \operatorname{dim}\left(V_{\zeta}\right) \leq \rho-s-1 \\
& n+s-\rho+1 \geq \operatorname{dim}\left(S_{x, y ; v}\right) \geq n+s-\rho .
\end{aligned}
$$

Suppose the right hand side values hold in (9). This implies that, for $z \in D_{\zeta}$ general, one has

$$
\left\langle T_{D_{\zeta}, z}, W\right\rangle=\left\langle T_{X, z}, W\right\rangle
$$

and this space projects down from $W$ to $T_{V_{\zeta}, v}$, with $v=\pi_{W}(z)$. Hence

$$
T_{X, z} \subseteq T:=\left\langle T_{V_{\zeta}, v}, W\right\rangle .
$$

One has again $\operatorname{dim}(T)=\rho$ and $T$ is tangent to $X$ along $S_{\zeta ; v}$. Then

$$
\rho \geq n+\operatorname{dim}\left(S_{\zeta ; v}\right)=2 n+s-\rho=4 n+1-\rho
$$

because $s=2 n+1$. Since $\rho \leq r-1$ we deduce

$$
n \leq \frac{2 r-3}{4} .
$$

On the other hand we have (1), which, together with (10) leads to a contradiction if $r \geq 6$. On the other hand if $r \leq 5$ there is no integer $n$ verifying both (10) and (1). In any event we have a contradiction.

If the right hand side equalities hold in (9), then we can repeat the same argument as above, which can now be left to the reader. This concludes the proof of Theorem 1.1.

Acknowledgements Open access funding provided by Università degli Studi di Roma Tor Vergata within the CRUI-CARE Agreement. The author is a member of GNSAGA of INdAM. He acknowledges the MIUR Excellence Department Project awarded to the Department of Mathematics, University of Rome Tor Vergata, CUP E83C18000100006. The author wishes to thank the referee for the careful reading of the paper and for appropriate remarks and suggestions.

Open Access This article is licensed under a Creative Commons Attribution 4.0 International License, which permits use, sharing, adaptation, distribution and reproduction in any medium or format, as long as you give appropriate credit to the original author(s) and the source, provide a link to the Creative Commons licence, and indicate if changes were made. The images or other third party material in this article are included in the article's Creative Commons licence, unless indicated otherwise in a credit line to the material. If material is not included in the article's Creative Commons licence and your intended use is not permitted by statutory regulation or exceeds the permitted use, you will need to obtain permission directly from the copyright holder. To view a copy of this licence, visit http://creativecommons.org/licenses/by/4.0/.

\section{References}

1. Chiantini, L., Ciliberto, C.: On the dimension of secant varieties. J. Eur. Math. Soc. 12(5), 1267-1291 (2010)

2. Ciliberto, C.: Attualità dei contributi di Alessandro Terracini su alcuni aspetti proiettivo-differenziali della geometria algebrica. (2020) (To appear)

3. Fulton, W., Lazarsfeld, R.: Connectivity and its applications in algebraic geometry. Algebraic Geometry (Chicago, IL, 1980). Lecture Notes in Mathematics, vol. 862, pp. 26-92. Springer, Berlin (1981)

4. Lopez, A., Ran, Z.: On the irreducibility of secant cones and an application to linear normality. Duke Math. J. 117(3), 389-401 (2003) 
5. Terracini, A.: Los $S_{2}$ osculadores a las curvas de una varieded y nueva caracterización de una clase de variedades. Rev. Mat. Fis. Tucuman 3, 317-339 (1942)

Publisher's Note Springer Nature remains neutral with regard to jurisdictional claims in published maps and institutional affiliations. 